EPJ Web of Conferences 43, 04004 (2013)

DOI: $10.1051 /$ epjconf/20134304004

(C) Owned by the authors, published by EDP Sciences, 2013

\title{
Pulsations driven by the $\epsilon$-mechanism in post-merger remnants: First results
}

\author{
M.M. Miller Bertolami ${ }^{1,2,3, a}$, A.H. Córsico ${ }^{1,2}$, X. Zhang ${ }^{4}$, L.G. Althaus ${ }^{1,2}$ \\ and C.S. Jeffery ${ }^{4,5}$ \\ ${ }^{1}$ Facultad de Ciencias Astronómicas y Geofísicas, Universidad Nacional de La Plata, \\ Paseo del Bosque s/n, 1900 La Plata, Argentina \\ ${ }^{2}$ CONICET, Paseo del Bosque s/n, 1900 La Plata, Argentina \\ ${ }^{3}$ Max-Planck-Institut für Astrophysik, Karl-Schwarzschild-Str. 1, 85748 Garching, Germany \\ ${ }^{4}$ Armagh Observatory, College Hill, Armagh BT61 9DG, UK \\ ${ }^{5}$ School of Physics, Trinity College Dublin, Dublin 2, Ireland
}

\begin{abstract}
Helium-rich subdwarfs are a rare subclass of hot subdwarf stars which constitute a small and inhomogeneous group showing varying degrees of helium enrichment. Only one star, LS IV ${ }^{\circ} 14116$ has been found to show multiperiodic luminosity variations. The variability of $\operatorname{LS} \mathrm{IV}^{\circ} 14116$ has been explained as the consequence of nonradial g-mode oscillations, whose excitation is difficult to understand within the frame of the standard $\kappa$-mechanism driving pulsations in sdBV stars. In a recent study, we have proposed that the pulsations of LS IV ${ }^{\circ} 14116$ might be driven through the $\epsilon$-mechanism acting in unstable Heburning zones in the interior of the star, that appear before the quiescent He-burning phase. One of the few accepted scenarios for the formation of He-rich subdwarfs is the merger of two He-core white dwarfs. As part of this project, we present a study of the $\epsilon$-mechanism in post-merger remnants, and discuss the results in the light of the pulsations exhibited by LS IV ${ }^{\circ} 14116$.
\end{abstract}

\section{INTRODUCTION}

About $5 \%$ of all hot subdwarf stars ( $\mathrm{sdB}, \mathrm{sdO})$ show helium $(\mathrm{He})$-enriched surface abundances (He-sdB, He-sdO). While most normal hydrogen $(\mathrm{H})$-rich subdwarfs are supposed to be low mass core He-burning stars with atmospheres dominated by $\mathrm{H}$ due to the action of gravitational settling, the evolutionary status of the He-rich subclass is less clear. He-rich subdwarfs have been suggested to be the result of either double He white dwarf (HeWD) mergers [1] or late helium core flashes (a.k.a. hot-flashers [2]). In fact, it is by no means clear whether intermediate and extreme He-rich sdB stars form a single class of objects or not [3]. Regardless of the particular scenario proposed for their formation, it is accepted that some of these stars are still contracting towards the He-core burning phase (EHB), as otherwise gravitational settling of the remaining $\mathrm{H}$ would have turned the star into a H-rich sdB star.

Two families of pulsators have been discovered within the H-rich sdB stars: the rapid pulsators (sdBVr, [4]) discovered by [5], and the slow pulsators (sdBVs, [4]) discovered by [6]. While sdBVr stars show short pulsation periods $(\sim 80-400 \mathrm{~s})$ ascribed to radial modes and nonradial $p$-modes, sdBVs pulsations (with periods $\sim 2500-7000 \mathrm{~s}$ ) are associated to nonradial long period $g$-modes. Pulsations in both groups of variable stars have been explained by the action of the $\kappa$-mechanism due to the partial ionization of iron group elements in the outer layers, where these elements are enhanced by the action

\footnotetext{
ae-mail: mmiller@f caglp.unlp.edu.ar
}

This is an Open Access article distributed under the terms of the Creative Commons Attribution License 2.0, which permits unrestricted use, distribution, and reproduction in any medium, provided the original work is properly cited. 


\section{EPJ Web of Conferences}

of radiative levitation [7] [8]. While many sdBV stars are known, only one He-sdB star, LS IV-14¹16, has been found to be a pulsator [9] [10]. In fact, LS IV-14 ${ }^{\circ} 116$ is a very intriguing object: while its atmospheric parameters ( $T_{\text {eff }}$ and $g$ ) place it well within the sdBVr instability region [11] [12], it displays periods typical of the sdBVs family of pulsators [10] [12]. From a spectroscopic point of view, LS IV$14^{\circ} 116$ is also an intriguing object, showing a mild He-enrichment $\left(n_{\mathrm{He}}=0.16\right)$ and very high excesses of zirconium, yttrium and strontium in its atmosphere [13]. In this sense, both the He-enrichment and the heavy metal excesses might be related to the effects of ongoing diffusion before reaching diffusive equilibrium, thus placing the star in the pre-EHB phase. Up to now, the driving mechanism behind the long period pulsations of LS IV- $14^{\circ} 116$, as well as the absence of short period pulsations, remains a mystery and the star poses a challenge to the theory of stellar pulsations [14]. In this connection, the recent confirmation of both the multiperiodic variability and the $T_{\text {eff }}-g$ values for LS IV-14 116 [12] strongly increase the enigma. In a recent article [15] we have suggested that pulsations in LS IV-14 116 might be excited by the $\epsilon$-mechanism acting on unstable He-burning shells that take place before the beginning of the EHB. In the $\epsilon$-mechanism, the driving is due to the strong dependence of nuclear burning rates on temperature. In the layers where nuclear reactions take place, thermal energy is gained at compression by the enhancement of nuclear energy liberation, while the opposite happens during the expansion phase [16]. In particular, in [15] we have shown that the $\epsilon$-mechanism is able to excite pulsations in hot-flasher stellar models. Although only one hot-flasher sequence was analyzed in [15], it was shown that the $\epsilon$-mechanism is qualitatively able to reproduce the long period $(P>1000 \mathrm{~s})$ $g$-modes at the values of $\log g$ and $\log T_{\text {eff }}$ inferred for LS IV-14 116 . As post-merger models also undergo off-centered He-shell flashes before settling on the EHB [1] and given that the location of the burning shell is particularly relevant for the range of excited periods [15], it seems natural to explore the effect of the $\epsilon$-mechanism in post HeWD merger stellar models.

Interestingly enough, no star has been identified so far to be excited by the epsilon mechanism. Aside from our previous suggestion [15], the only possible exceptions are some oscillations in $\delta$ Scuti stars [17], PG 1159 stars [18] [19], and more recently, some very long periods of Rigel [20], but none of these have yet been confirmed. In this connection, the identification of the $\epsilon$-mechanism as the driving mechanism behind the lightcurve variations of LS IV-14 ${ }^{\circ} 116$ would offer the first evidence that the $\epsilon$-mechanism can indeed drive pulsations in stars.

\section{NUMERICAL METHODS AND INPUT PHYSICS}

Numerical models of 1D-post-merger structures were constructed by simulating the merger process as an accretion process of the material of the secondary on top of the primary (a He-WD model). Specifically most of our models are constructed with MESA stellar evolution code [21] following the recipe detailed in [22] for fast merger episodes in which the accretion rate is of $10^{4} \mathrm{M}_{\odot} / \mathrm{yr}$. Also some variants of the "composite merger" (see [22]) were computed. It is worth noting that nucleosynthesis is included during the accretion process. The composition of the accreted material was, by mass fraction, ${ }^{12} \mathrm{C}=6 \times 10^{-5},{ }^{14} \mathrm{~N}=1.27 \times 10^{-2},{ }^{16} \mathrm{O}=1.64 \times 10^{-3},{ }^{20} \mathrm{Ne}=1.88 \times 10^{-3}$ and ${ }^{24} \mathrm{Mg}=3.68 \times 10^{-3}$ for the $Z=0.02$ sequences and of ${ }^{12} \mathrm{C}=3.1 \times 10^{-5},{ }^{14} \mathrm{~N}=2.5 \times 10^{-3}$, ${ }^{16} \mathrm{O}=9.3 \times 10^{-5},{ }^{20} \mathrm{Ne}=3.9 \times 10^{-4}$ and ${ }^{24} \mathrm{Mg}=8.0 \times 10^{-4}$ for the $Z=0.004$ sequences. Hydrogen present in both the accretor and the accreted material was neglected. Evolution during the helium shell sub-flashes that follow the accretion process was followed with LPCODE stellar evolution code [23], which has been extensively used in the computation of He-flashes (e.g. [24] and [25]). The pulsational instability analysis presented in this work was carried out with the linear, nonradial, nonadiabatic pulsation code described in detail in [19] and references therein, in which the $\epsilon$-mechanism for driving pulsations in the He-burning regions is fully taken into account. As described in [15] the "frozen-in convection" approximation was asssumed because the timescales of the pulsations are usually much shorter than the convective turnover times. 


\section{Ageing Low Mass Stars: From Red Giants to White Dwarfs}

Table 1. Global properties of the merger sequences studied in this work compared with those of LS IV- $14^{\circ} 116$ and the hot-flasher sequence of [15].

\begin{tabular}{lllll}
\hline & $\begin{array}{l}\text { Stellar Mass } \\
\left(\mathrm{M}_{\odot}\right)\end{array}$ & $\begin{array}{l}\text { Initial Metallicity } \\
\left(\mathrm{Z}_{0}\right)\end{array}$ & $\begin{array}{l}\text { Range of } \\
\text { excited periods }\end{array}$ & $\begin{array}{l}\mathrm{H} \text { and He } \\
\text { abundances by mass } \\
\text { fraction }\end{array}$ \\
\hline $0.25 \mathrm{M}_{\odot}+0.25 \mathrm{M}_{\odot}$ & $0.50 \mathrm{M}_{\odot}$ & 0.02 & $<3700 \mathrm{~s}$ & without $\mathrm{H}$ \\
$0.20 \mathrm{M}_{\odot}+0.20 \mathrm{M}_{\odot}$ & $0.40 \mathrm{M}_{\odot}$ & 0.02 & $<2600 \mathrm{~s}$ & without $\mathrm{H}$ \\
$0.25 \mathrm{M}_{\odot}+0.15 \mathrm{M}_{\odot}$ & $0.40 \mathrm{M}_{\odot}$ & 0.02 & $<2700 \mathrm{~s}$ & without $\mathrm{H}$ \\
$0.30 \mathrm{M}_{\odot}+0.10 \mathrm{M}_{\odot}$ & $0.40 \mathrm{M}_{\odot}$ & 0.02 & $<2500 \mathrm{~s}$ & without $\mathrm{H}$ \\
$0.25 \mathrm{M}_{\odot}+0.19 \mathrm{M}_{\odot}$ & $0.44 \mathrm{M}_{\odot}$ & 0.004 & $<2250 \mathrm{~s}$ & without $\mathrm{H}$ \\
$0.35 \mathrm{M}_{\odot}+0.09 \mathrm{M}_{\odot}$ & $0.44 \mathrm{M}_{\odot}$ & 0.004 & $<3440 \mathrm{~s}$ & without H \\
$0.25 \mathrm{M}_{\odot}+0.25 \mathrm{M}_{\odot}$ & $0.50 \mathrm{M}_{\odot}$ & 0.02 & $<3700 \mathrm{~s}$ & $\mathrm{H}=0.456, \mathrm{He}=0.509$ \\
$0.25 \mathrm{M}_{\odot}+0.15 \mathrm{M}_{\odot}$ & $0.40 \mathrm{M}_{\odot}$ & 0.02 & $<2700 \mathrm{~s}$ & $\mathrm{H}=0.456, \mathrm{He}=0.517$ \\
\hline Shallow Mixing [15] & $0.47378 \mathrm{M}_{\odot}$ & 0.02 & $<2200 \mathrm{~s}$ & $\mathrm{H}=0.382, \mathrm{He}=0.598$ \\
$\mathbf{L S ~ I V - 1 4} \mathbf{1 1 6}[13]$ & - & - & $1954 \mathrm{~s}-5084 \mathrm{~s}$ & $\mathrm{H}=0.544, \mathrm{He}=0.452$ \\
\hline
\end{tabular}

\subsection{Computed sequences}

In order to study the excitation of $g$-modes through the $\epsilon$-mechanism acting on the He-subflashes, and also to allow for comparisons with LS IV-14 ${ }^{\circ} 116$, we analysed the stability of modes with periods between $500 \mathrm{~s}$ and $6000 \mathrm{~s}$. Modes were analysed every 5 stellar evolution timesteps during the whole pre-EHB phase for all evolutionary sequences. The analysed sequences are detailed in Table 1 and the location of the sequences in the $\log g$ - $\log T_{\text {eff }}$ diagram is shown in Fig. 1. In addition, we analysed two sequences for which we added artificially a $\mathrm{H}$-rich envelope with a $\mathrm{H} / \mathrm{He}$ mixture similar to that inferred in LS IV-14 116 . In order to maximize the effect of the presence of $\mathrm{H}, \mathrm{H}$ was added as deep as possible without reaching layers with temperatures high enough to burn $\mathrm{H}\left(\log \left(1-m(r) / M_{\text {star }}\right) \sim-3.85\right)$. As will be shown, when comparing the $\log g-\log T_{\text {eff }}$ of theoretical sequences and real stars, the total amount of $\mathrm{H}$ in the envelope should be taken into account.

\section{DISCUSSION AND CONCLUSIONS}

From Fig. 1 it is clear that more massive models evolve during the He-core flashes at higher temperatures than less massive ones. This is not unexpected as pure helium structures will settle on the so-called He main sequence [26] which lies at higher temperatures for more massive models. Consequently the location of our sequences of higher masses is unable to fit the observed parameters of LS IV-14 116 . It should be noted, however, that when $\mathrm{H}$ is added in the envelope of the theoretical sequences, tracks are shifted to lower temperatures and gravities, closer to the observed location of LS IV-14 116 (see top panels of Fig. 1). In the second and third row panels of Fig. 1, the evolution of the lower mass mergers analysed in this work is shown $\left(M_{\text {final }}=0.4 \mathrm{M}_{\odot}\right)$. As can be seen, lower mass models evolve in the pre$\mathrm{HB}$ at lower temperatures (and through wider loops) than the more massive ones attaining temperatures similar to those of LS IV- $14^{\circ} 116$. In addition, it can be seen that the surface gravities of the sequences depend on how the final mass is attained, with mergers with initially more massive primary stars (i.e. lower accreted masses) evolving at lower gravities.

As shown in Table 1 and Fig. 2 the $\epsilon$-mechanism in some of the more massive post-merger models is able to excite periods as long as $3700 \mathrm{~s}$, significantly longer than those predicted by the hot-flasher model studied in [15], making the predicted periods of the unstable modes closer to those of LS IV$14^{\circ} 116$. It should be noted however, that the formally unstable phase of the more massive sequence during the first flash (top panel, Fig. 2) is too short, with the shortest e-folding times very similar to 

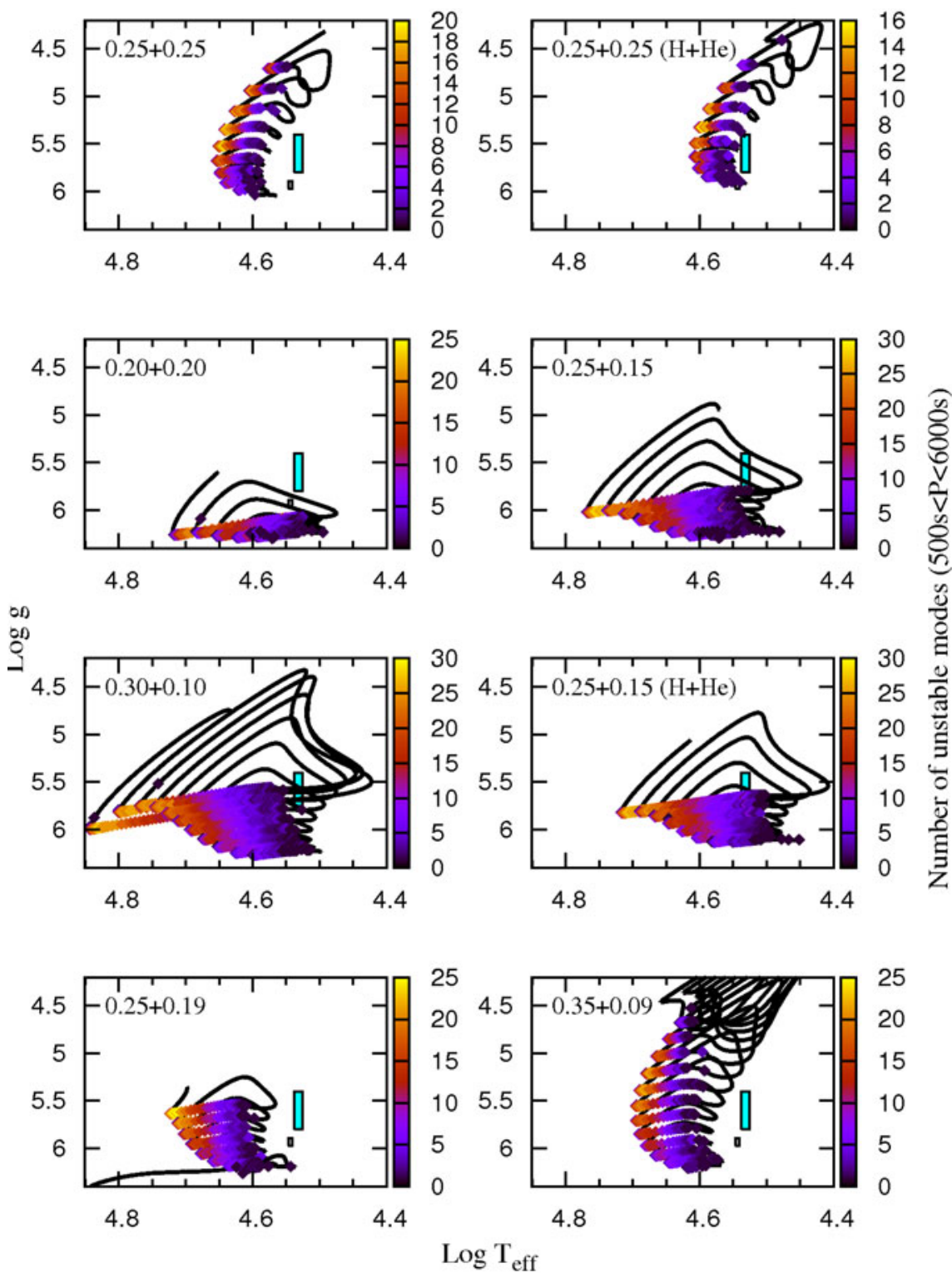

Figure 1. Location in the $\log g-\log T_{\text {eff }}$ of the post-merger sequences studied in this work. Thick color points indicate the location of models for which pulsations driven by the $\epsilon$-mechanism have been found with periods in the studied range. Color coding indicates the number of unstable modes found. Note that in all the sequences unstable modes are found during the fast redward evolution during the He-shell flashes. Cyan and grey boxes indicate the derived atmosphere parameters of LS IV- $14^{\circ} 116$ derived by [13] and [12], respectively.

the length of the instability phase $(\sim 100 \mathrm{yr})$. Then, although that sequence displays formally unstable modes as long as $3700 \mathrm{~s}$ they will not be observable. The situation is different for the less massive sequences (middle and bottom panels of Fig. 2), for which the instability phase is longer than the e-folding times, and thus pulsations driven by the $\epsilon$-mechanism should be observed. It is particularly worth noting that for the $0.25 \mathrm{M}_{\odot}+0.15 \mathrm{M}_{\odot}$ sequence with $\mathrm{H}$ in the envelope (third row, right panel in Fig. 1), the remnant evolves through the observed location of LS IV-14 116 during the first three 
Ageing Low Mass Stars: From Red Giants to White Dwarfs
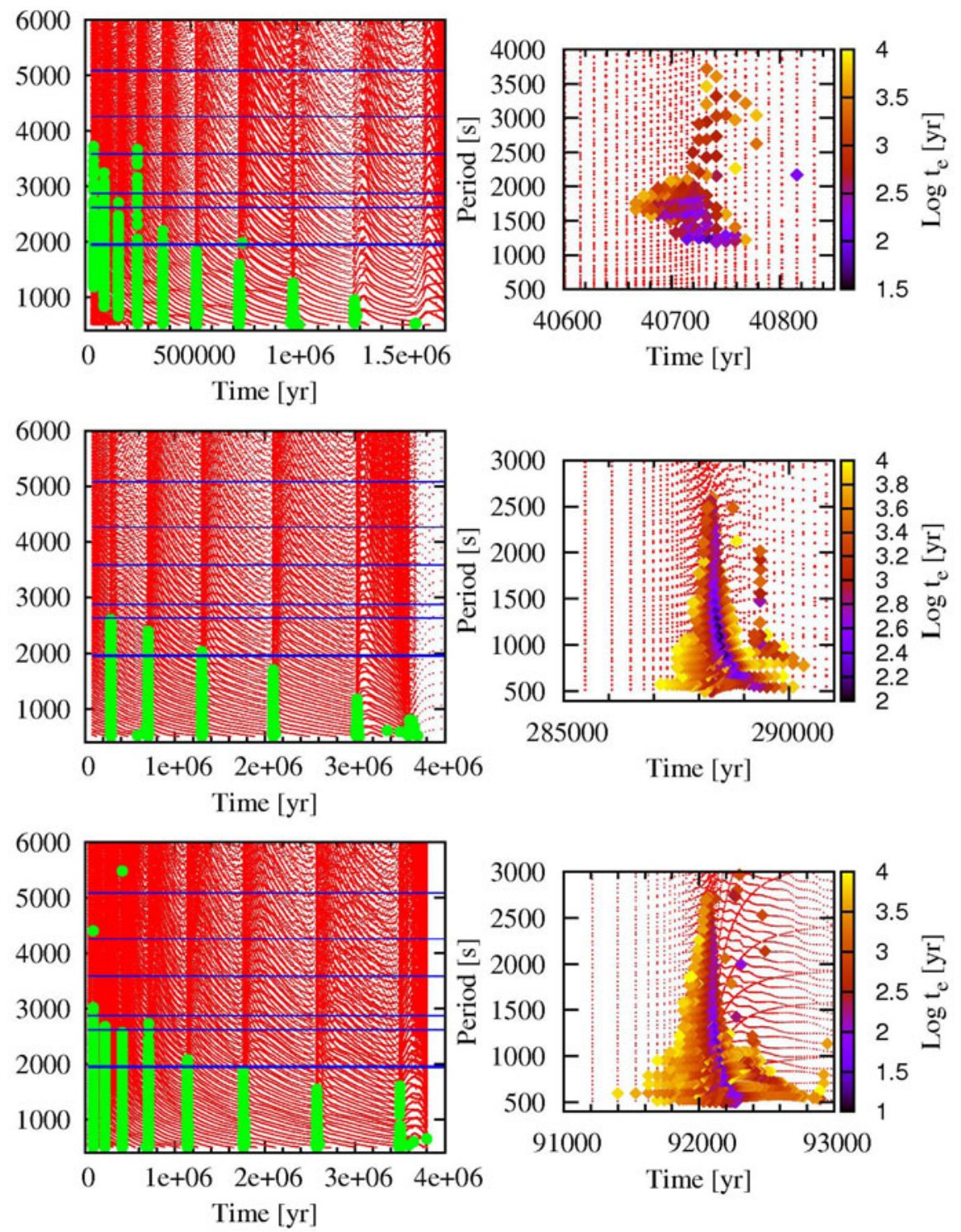

Figure 2. Left: Evolution of the periods of the normal modes during the pre-EHB evolution. Excited periods through the $\epsilon$-mechanism are marked with green thick points and compared with the observed modes of LS IV- $14^{\circ} 116$ (blue horizontal lines). Right: Same as left but only during the first (and most violent) flash. Color coding indicates the value of the e-folding time of the unstable periods. The sequences plotted are the $0.25 \mathrm{M}_{\odot}+0.25 \mathrm{M}_{\odot}$ merger (top), the $0.20 \mathrm{M}_{\odot}+0.20 \mathrm{M}_{\odot}$ merger (middle) and the $0.25 \mathrm{M}_{\odot}+0.15 \mathrm{M}_{\odot}$ merger with an added hydrogen envelope (bottom).

flashes, showing during this stage excited periods as long as the two shortest periods observed in LS IV$14^{\circ} 116$ ( $P=1953.74,2620.27 \mathrm{~s}$ [12]). In particular, the range of excited periods in the first five flashes is able to reproduce the higher amplitude mode of LS IV-14 ${ }^{\circ} 116(P=1953.74 \mathrm{~s})$. Still, the excited modes of all our computed sequences have periods too short to explain the longest periods observed in LS IV-14 $116(P>4000 \mathrm{~s})$. It should be noted, however, that the merger models we have adopted in this study are based on some simplifying assumptions, and thus more detailed merger models should be 
explored before making a final statement about the origin of LS IV $-14^{\circ} 116$ and the mechanism behind its pulsations. In particular, it is worth noting that the thermal structure of post-merger models is sensitive to the details of the merger process and on the relative masses of the primary and secondary stars [27]. A more realistic treatment of the merger process might lead to thermal structures significantly different and thus to a shift in the location of the He-core flashes, altering the range of excited periods. One interesting feature predicted by the present explanation of the pulsations observed in LS IV-14 116 is the large magnitude of the rate of period changes of the modes. In fact, as the structure changes very fast during the helium core flashes, then the predicted period change can be as high as a few seconds per year. The determination of large value in the rate of period changes would be a strong indication that LS IV-14 116 is in a fast evolutionary stage. In this connection it might be worth noting that the value of the determined period of the $\sim 2870 \mathrm{~s}$ mode of LS IV- $14^{\circ} 116$ differs in about $2 \mathrm{~s}$ from the observations done in 2004 [10] and the determinations of 2010 [12], which is somewhat larger than the quoted error bars $(\sigma<1 \mathrm{~s})$.

Despite the particular origin of $\operatorname{LS} \operatorname{IV}-14^{\circ} 116$, and it is by no means obvious whether it is a postmerger object or not, our analysis suggests that post HeWD+HeWD merger remnants should go through a phase in which pulsations are driven by the $\epsilon$-mechanism. Due to the short duration of the unstable phases, only $\sim 1$ every 100 pre-EHB objects should be pulsators. A more detailed exploration of the parameter space of both the merger and hot-flasher scenarios to understand the pulsations excited in LS IV-14 116 will be performed in forthcoming works. We expect that future work will help to distinguish between the possible evolutionary channels for LS IV-14 116.

M3B thanks the organizers of the "40th Liege International Astrophysical Colloquium" for the financial assistance that helped him to attend the conference. This research was supported by PIP 112-200801-00940 from CONICET and PICT-2010-0861 from ANCyT.

\section{References}

[1] H. Saio, C.S. Jeffery, MNRAS 313, 671 (2000)

[2] T.M. Brown, A.V. Sweigart, T. Lanz, W.B. Landsman, I. Hubeny, ApJ 562, 368 (2001)

[3] C.S. Jeffery, in The 61th Fujihara Seminar, edited by H. Shibahashi, M. Takata, \& A. Lynas-Gray (2012), Vol. 462 of Astronomical Society of the Pacific Conference Series, pp. 47-+

[4] D. Kilkenny, G. Fontaine, E.M. Green, S. Schuh, Information Bulletin on Variable Stars 5927, 1 (2010)

[5] D. Kilkenny, C. Koen, D. O’Donoghue, R.S. Stobie, MNRAS 285, 640 (1997)

[6] E.M. Green, G. Fontaine, M.D. Reed, K. Callerame, I.R. Seitenzahl, B.A. White, E.A. Hyde, R. Østensen, O. Cordes, P. Brassard et al., ApJL 583, L31 (2003)

[7] S. Charpinet, G. Fontaine, P. Brassard, P. Chayer, F.J. Rogers, C.A. Iglesias, B. Dorman, ApJL 483, L123+ (1997)

[8] G. Fontaine, P. Brassard, S. Charpinet, E.M. Green, P. Chayer, M. Billères, S.K. Randall, ApJ 597, 518 (2003)

[9] A. Ahmad, C.S. Jeffery, J.E. Solheim, R. Østensen, Ap\&SS 291, 435 (2004)

[10] A. Ahmad, C.S. Jeffery, A\&A 437, L51 (2005)

[11] N. Naslim, C.S. Jeffery, A. Ahmad, N.T. Behara, T. Şahìn, MNRAS 409, 582 (2010)

[12] E.M. Green, B. Guvenen, C.J. O’Malley, C.J. O’Connell, B.P. Baringer, A.S. Villareal, T.M. Carleton, G. Fontaine, P. Brassard, S. Charpinet, ApJ 734, 59 (2011)

[13] N. Naslim, C.S. Jeffery, N.T. Behara, A. Hibbert, MNRAS 412, 363 (2011) 
[14] G. Fontaine, P. Brassard, S. Charpinet, E.M. Green, P. Chayer, S.K. Randall, V. van Grootel, in Hot Subdwarf Stars and Related Objects, edited by U. Heber, C. S. Jeffery, \& R. Napiwotzki (2008), Vol. 392 of Astronomical Society of the Pacific Conference Series, pp. 231-+

[15] M.M. Miller Bertolami, A.H. Córsico, L.G. Althaus, ApJL 741, L3 (2011)

[16] W. Unno, Y. Osaki, H. Ando, H. Saio, H. Shibahashi, Nonradial oscillations of stars (1989)

[17] G. Lenain, R. Scuflaire, M.A. Dupret, A. Noels, Communications in Asteroseismology 147, 93 (2006)

[18] S.D. Kawaler, D.E. Winget, C.J. Hansen, I. Iben, Jr., ApJL 306, L41 (1986)

[19] A.H. Córsico, L.G. Althaus, M.M. Miller Bertolami, J.M. González Pérez, S.O. Kepler, ApJ 701, 1008 (2009)

[20] E. Moravveji, A. Moya, E.F. Guinan, ApJ 749, 74 (2012)

[21] B. Paxton, L. Bildsten, A. Dotter, F. Herwig, P. Lesaffre, F. Timmes, ApJS 192, 3 (2011)

[22] X. Zhang, C.S. Jeffery, MNRAS 419, 452 (2012)

[23] L.G. Althaus, A.M. Serenelli, J.A. Panei, A.H. Córsico, E. García-Berro, C.G. Scóccola, A\&A 435, 631 (2005)

[24] M.M. Miller Bertolami, L.G. Althaus, A.M. Serenelli, J.A. Panei, A\&A 449, 313 (2006)

[25] M.M. Miller Bertolami, L.G. Althaus, K. Unglaub, A. Weiss, A\&A 491, 253 (2008)

[26] R. Kippenhahn, A. Weigert, Stellar Structure and Evolution (1994)

[27] P. Lorén-Aguilar, J. Isern, E. García-Berro, A\&A 500, 1193 (2009) 